\title{
ON THE OSCILLATION OF A CLASS OF DAMPED FRACTIONAL DIFFERENTIAL EQUATIONS
}

\author{
ERCAN TUNÇ AND OSMAN TUNÇ
}

Received 07 October, 2015

Abstract. Using Riccati type transformations, the authors establish some new oscillation criteria for the fractional differential equation

$$
\left(D_{0+\alpha}^{1+\alpha} y\right)(t)+p(t)\left(D_{0+}^{\alpha} y\right)(t)+q(t) f(G(t))=0, \quad t>0,
$$

where $D_{0+}^{\alpha} y$ is the Riemann-Liouville fractional derivative of order $\alpha$ of $y, G(t)=\int_{0}^{t}(t-s)^{-\alpha} y(s) d s$, and $\alpha \in(0,1)$. Examples are provided to illustrate the relevance of the results.

2010 Mathematics Subject Classification: 34A08; 34C10

Keywords: oscillatory solutions, fractional differential equation, integral averaging technique, Riccati transformation

\section{INTRODUCTION}

In this paper we are concerned with the oscillatory nature of the fractional differential equation with damping

$$
\left(D_{0+}^{1+\alpha} y\right)(t)+p(t)\left(D_{0+}^{\alpha} y\right)(t)+q(t) f(G(t))=0, \quad t \geq t_{0}>0,
$$

where $\alpha \in(0,1)$ is a constant, $D_{0+}^{\alpha} y$ is the $\alpha$-th Riemann-Liouville fractional derivative of $y$, and $G(t)=\int_{0}^{t}(t-s)^{-\alpha} y(s) d s$.

We assume throughout this paper that the following conditions hold:

(C1) $p:\left[t_{0}, \infty\right) \rightarrow R$ is a continuous function with $p(t)<0$;

(C2) $q:\left[t_{0}, \infty\right) \rightarrow R$ is a continuous function with $q(t) \geq 0$;

(C3) $f: R \rightarrow R$ is a continuous function with $u f(u)>0$ for $u \neq 0$ and there exists a constant $K>0$ such that $\frac{f(u)}{u} \geq K$ for all $u \neq 0$.

A nontrivial function $y(t)$ is said to be a solution of equation (1.1) if $y(t) \in$ $C((0, \infty), R), \int_{0}^{t}(t-s)^{-\alpha} y(s) d s \in C^{1}((0, \infty), R), D_{0+}^{\alpha} y \in C^{1}((0, \infty), R)$, and satisfies $(1.1)$ on $(0, \infty)$. Our attention is restricted to those solutions of $(1.1)$ which 
exist on $(0, \infty)$ and satisfy $\sup \left\{|y(t)|: t \geq t_{*}\right\}>0$ for any $t_{*}>0$. Such a solution of equation (1.1) is said to be oscillatory if it is neither eventually positive nor eventually negative, and it is nonoscillatory otherwise. Equation (1.1) is said to be oscillatory if all its solutions are oscillatory.

Differential equations of fractional orders appear more and more frequently in various research areas and applications in diverse fields of science and engineering. Readers are referred to the monographs $[2,7,8,10]$ for the theory and applications of fractional calculus.

The problem of determining the oscillation of solutions of various equations like ordinary differential equations, difference equations, and functional differential equations has been a very active area of research in the last few decades, for example, see $[1,5,11,12]$ and the references therein.

However, to the best of our knowledge, only a few papers considered the oscillatory behavior of fractional differential equations. We refer the reader to $[3,6,9,13]$ and the references therein for problems involving Riemann-Liouville fractional derivatives; for the problems involving Caputo fractional derivatives, the reader is referred to $[4,6]$ and the included references.

The motivation for the present work has been inspired basically by the papers of Chen [3], Prakash et.al [9], and the cited papers in the references. By using Riccati type transformations and the integral averaging technique, we establish some new sufficient conditions which guarantee the oscillation of solutions of equation (1.1). It is therefore hoped that the present paper will contribute to the studies in oscillatory behavior of solutions of fractional differential equations with damping. Finally, some examples are given to illustrate our results.

\section{Preliminaries}

In this section, we introduce some basic notations, definitions, and preliminary facts from fractional calculus; see $[2,7,8,10]$ for additional details.

Definition 1. The Riemann-Liouville fractional integral of order $\beta>0$ for a function $y:(0, \infty) \rightarrow R$ is defined by

$$
\left(I_{0+}^{\beta} y\right)(t):=\frac{1}{\Gamma(\beta)} \int_{0}^{t}(t-s)^{\beta-1} y(s) d s, \quad t>0,
$$

provided the right side exists pointwise on $(0, \infty)$, where $\Gamma$ is the Gamma function. 
ON THE OSCILLATION OF A CLASS OF DAMPED FRACTIONAL DIFFERENTIAL EQUATIONS 649

Definition 2. The Riemann-Liouville fractional derivative of order $\beta>0$ for a function $y:(0, \infty) \rightarrow R$ is defined by

$$
\left(D_{0+}^{\beta} y\right)(t):=\frac{1}{\Gamma(n-\beta)} \frac{d^{n}}{d t^{n}} \int_{0}^{t}(t-s)^{n-\beta-1} y(s) d s, \quad t>0,
$$

provided the righ-hand side exists, where $n=[\beta]+1$ and $[\beta]$ denotes the integer part of $\beta$.

To obtain our results in this paper, we need the following two lemmas.

Lemma 1. Let $y$ be solution of (1.1), and

$$
G(t):=\int_{0}^{t}(t-s)^{-\alpha} y(s) d s \quad \text { for } \alpha \in(0,1), \quad t>0 .
$$

Then

$$
G^{\prime}(t)=\Gamma(1-\alpha)\left(D_{0+}^{\alpha} y\right)(t) .
$$

Proof. Differentiating (2.3) and using (2.2), we have, for $\alpha \in(0,1)$ and $t>0$,

$$
\begin{aligned}
G^{\prime}(t) & =\frac{d}{d t} \int_{0}^{t}(t-s)^{-\alpha} y(s) d s \\
& =\Gamma(1-\alpha) \frac{1}{\Gamma(1-\alpha)} \frac{d}{d t} \int_{0}^{t}(t-s)^{-\alpha} y(s) d s \\
& =\Gamma(1-\alpha)\left(D_{0+}^{\alpha} y\right)(t) .
\end{aligned}
$$

This completes the proof proof of Lemma 1.

Lemma 2. Let $\alpha \in(0,1)$, and $t>0$. If $y$ is a solution of $(1.1)$, then $\left(D_{0+}^{1+\alpha} y\right)(t)=$ $\left(D_{0+}^{\alpha} y\right)^{\prime}(t)$.

Proof. From (2.2) and $n=[\beta]+1=[1+\alpha]+1=[\alpha]+1+1=2$, we obtain

$$
\begin{aligned}
\left(D_{0+}^{1+\alpha} y\right)(t) & =\frac{1}{\Gamma(2-\alpha-1)} \frac{d^{2}}{d t^{2}} \int_{0}^{t}(t-s)^{2-\alpha-1-1} y(s) d s \\
& =\frac{1}{\Gamma(1-\alpha)} \frac{d^{2}}{d t^{2}} \int_{0}^{t}(t-s)^{-\alpha} y(s) d s
\end{aligned}
$$




$$
\begin{aligned}
& =\frac{d}{d t}\left(\frac{1}{\Gamma(1-\alpha)} \frac{d}{d t} \int_{0}^{t}(t-s)^{-\alpha} y(s) d s\right) \\
& =\frac{d}{d t}\left(D_{0+}^{\alpha} y\right)(t)=\left(D_{0+}^{\alpha} y\right)^{\prime}(t) .
\end{aligned}
$$

This completes the proof of Lemma 2.

\section{MAIN RESUlTS}

Theorem 1. Suppose that (C1)-(C3) hold. If

$$
\lim _{t \rightarrow \infty}\left[\frac{1}{4 \Gamma(1-\alpha)} \int_{t_{0}}^{t}\left(4 \Gamma(1-\alpha) K q(s)-p^{2}(s)\right) d s\right]=\infty,
$$

then any solution of the equation (1.1) is oscillatory.

Proof of Theorem 1. Let $y(t)$ be a nonoscilatory solution of equation (1.1). Without loss of generality, we may assume that $y$ is an eventually positive solution of (1.1). Then, there exists $t_{1} \in\left[t_{0}, \infty\right)$ such that $y(t)>0$ and $G(t)>0$ for $t \geq t_{1}$. Define the function $w$ by

$$
w(t)=-\frac{\left(D_{0+}^{\alpha} y\right)(t)}{G(t)}, \quad \text { for } t \geq t_{1} .
$$

Then $w(t)$ is well defined and satisfies the inequality

$$
\begin{aligned}
w^{\prime}(t) & =-\left[\frac{\left(\left(D_{0+}^{\alpha} y\right)(t)\right)^{\prime} G(t)-\left(D_{0+}^{\alpha} y\right)(t) G^{\prime}(t)}{G^{2}(t)}\right] \\
& =-\left[\frac{\left(D_{0+}^{1+\alpha} y\right)(t)}{G(t)}-\frac{\Gamma(1-\alpha)\left(\left(D_{0+}^{\alpha} y\right)(t)\right)^{2}}{G^{2}(t)}\right] \\
& =p(t) \frac{\left(D_{0+}^{\alpha} y\right)(t)}{G(t)}+q(t) \frac{f(G(t))}{G(t)}+\Gamma(1-\alpha)\left(\frac{\left(D_{0+}^{\alpha} y\right)(t)}{G(t)}\right)^{2} \\
& \geq-p(t) w(t)+K q(t)+\Gamma(1-\alpha) w^{2}(t) .
\end{aligned}
$$

Integrating (3.2) from $t_{1}$ to $t$, we obtain

$$
\begin{aligned}
w(t) & \geq w\left(t_{1}\right)+\int_{t_{1}}^{t}\left[\Gamma(1-\alpha) w^{2}(s)-p(s) w(s)+K q(s)\right] d s \\
& =w\left(t_{1}\right)+\Gamma(1-\alpha) \int_{t_{1}}^{t}\left(w(s)-\frac{p(s)}{2 \Gamma(1-\alpha)}\right)^{2} d s
\end{aligned}
$$


ON THE OSCILLATION OF A CLASS OF DAMPED FRACTIONAL DIFFERENTIAL EQUATIONS 651

$$
+\frac{1}{4 \Gamma(1-\alpha)} \int_{t_{1}}^{t}\left(4 \Gamma(1-\alpha) K q(s)-p^{2}(s)\right) d s .
$$

In view of (3.1), there exists $t_{2} \geq t_{1}$, such that

$$
w(t)>\Gamma(1-\alpha) \int_{t_{2}}^{t}\left(w(s)-\frac{p(s)}{2 \Gamma(1-\alpha)}\right)^{2} d s \text { for } t \geq t_{2} .
$$

If we set

$$
H(t)=\Gamma(1-\alpha) \int_{t_{2}}^{t}\left(w(s)-\frac{p(s)}{2 \Gamma(1-\alpha)}\right)^{2} d s,
$$

then we have $w(t)>H(t)>0$ for $t \geq t_{2}$.

From (3.4) and using the fact that $p(t)<0$, we can easily see that

$$
H^{\prime}(t)=\Gamma(1-\alpha)\left(w(t)-\frac{p(t)}{2 \Gamma(1-\alpha)}\right)^{2}>\Gamma(1-\alpha) w^{2}(t)>\Gamma(1-\alpha) H^{2}(t),
$$

and so we obtain

$$
\Gamma(1-\alpha)<\frac{H^{\prime}(t)}{H^{2}(t)} \text { for } t \geq t_{2}
$$

Integrating both sides of this inequality from $t_{2}$ to $t$, we obtain that

$$
\Gamma(1-\alpha) \int_{t_{2}}^{t} d s<\frac{1}{H\left(t_{2}\right)}-\frac{1}{H(t)}<\frac{1}{H\left(t_{2}\right)} .
$$

Letting $t \rightarrow \infty$ in the above inequality gives

$$
\lim _{t \rightarrow \infty} \Gamma(1-\alpha) \int_{t_{2}}^{t} d s<\frac{1}{H\left(t_{2}\right)},
$$

but this is not correct. This completes the proof of the theorem.

Theorem 2. Let conditions $(C 1)-(C 3)$ hold. Assume that there exists a positive function $g \in C^{1}\left[t_{0}, \infty\right)$ such that

$$
\lim _{t \rightarrow \infty} \int_{t_{0}}^{t} \frac{\Gamma(1-\alpha)}{g(s)} d s=\infty
$$

and

$$
\lim _{t \rightarrow \infty}\left[-\frac{1}{4 \sqrt{\Gamma(1-\alpha)}} \int_{t_{0}}^{t} \Psi(s) d s+\frac{g^{\prime}(s)}{2 \sqrt{\Gamma(1-\alpha)}}\right]=\infty
$$


where

$$
\Psi(s)=p^{2}(s) g(s)+\frac{\left(g^{\prime}(s)\right)^{2}}{g(s)}-2 p(s) g^{\prime}(s)-4 K \Gamma(1-\alpha) g(s) q(s),
$$

then any solution of the equation (1.1) is oscillatory.

Proof of Theorem 2. Let $y(t)$ be a nonoscilatory solution of equation (1.1). Without loss of generality we may assume that $y$ is an eventually positive solution of (1.1). Then, there exists $t_{1} \in\left[t_{0}, \infty\right)$ such that $y(t)>0$ and $G(t)>0$ for $t \geq t_{1}$. Define the function $w$ by

$$
w(t)=-g(t) \frac{\left(D_{0+}^{\alpha} y\right)(t)}{G(t)} \text { for } t \geq t_{1} .
$$

In view of conditions (C1)-(C3), from equation (1.1) we obtain

$$
\begin{aligned}
w^{\prime}(t) & =-g^{\prime}(t) \frac{\left(D_{0+}^{\alpha} y\right)(t)}{G(t)}-g(t)\left[\frac{\left(D_{0+}^{\alpha} y\right)(t)}{G(t)}\right]^{\prime} \\
& =\frac{g^{\prime}(t)}{g(t)} w(t)-g(t)\left[\frac{\left(\left(D_{0+}^{\alpha} y\right)(t)\right)^{\prime} G(t)-\left(D_{0+}^{\alpha} y\right)(t) G^{\prime}(t)}{G^{2}(t)}\right] \\
& =\frac{g^{\prime}(t)}{g(t)} w(t)-g(t)\left[\frac{\left(D_{0+}^{1+\alpha} y\right)(t)}{G(t)}-\frac{\Gamma(1-\alpha)\left(\left(D_{0+}^{\alpha} y\right)(t)\right)^{2}}{G^{2}(t)}\right] \\
& =\frac{g^{\prime}(t)}{g(t)} w(t)+g(t) p(t) \frac{\left(D_{0+}^{\alpha} y\right)(t)}{G(t)}+g(t) q(t) \frac{f(G(t))}{G(t)} \\
& +\Gamma(1-\alpha) g(t)\left(\frac{\left(D_{0+}^{\alpha} y\right)(t)}{G(t)}\right)^{2} \\
& \geq \frac{1}{g(t)}\left[\Gamma(1-\alpha) w^{2}(t)-g(t) p(t) w(t)+g^{\prime}(t) w(t)\right]+K g(t) q(t) .
\end{aligned}
$$

Taking

$$
H(t)=\sqrt{\Gamma(1-\alpha)} w(t)+\frac{1}{2 \sqrt{\Gamma(1-\alpha)}} g^{\prime}(t),
$$

we get from (3.8) that

$$
\begin{aligned}
w^{\prime}(t) & \geq \frac{1}{g(t)}\left[\left(H(t)-\frac{p(t) g(t)}{2 \sqrt{\Gamma(1-\alpha)}}\right)^{2}-\left(\frac{p(t) g(t)}{2 \sqrt{\Gamma(1-\alpha)}}\right)^{2}-\frac{\left(g^{\prime}(t)\right)^{2}}{4 \Gamma(1-\alpha)}\right. \\
& \left.+\frac{p(t) g(t) g^{\prime}(t)}{2 \Gamma(1-\alpha)}\right]+K g(t) q(t)
\end{aligned}
$$


ON THE OSCILLATION OF A CLASS OF DAMPED FRACTIONAL DIFFERENTIAL EQUATIONS 653

$$
\begin{aligned}
& =\frac{1}{g(t)}\left(H(t)-\frac{p(t) g(t)}{2 \sqrt{\Gamma(1-\alpha)}}\right)^{2} \\
& -\frac{1}{4 \Gamma(1-\alpha)}\left[p^{2}(t) g(t)+\frac{\left(g^{\prime}(t)\right)^{2}}{g(t)}-2 p(t) g^{\prime}(t)-4 K \Gamma(1-\alpha) g(t) q(t)\right] .
\end{aligned}
$$

Integrating both of sides of the above equation from $t_{1}$ to $t$, we obtain

$$
\begin{gathered}
w(t) \geq w\left(t_{1}\right)+\int_{t_{1}}^{t} \frac{1}{g(s)}\left(H(s)-\frac{p(s) g(s)}{2 \sqrt{\Gamma(1-\alpha)}}\right)^{2} d s \\
-\frac{1}{4 \Gamma(1-\alpha)} \int_{t_{1}}^{t}\left[p^{2}(s) g(s)+\frac{\left(g^{\prime}(s)\right)^{2}}{g(s)}-2 p(s) g^{\prime}(s)-4 K \Gamma(1-\alpha) g(s) q(s)\right] d s .
\end{gathered}
$$

Using (3.9) in (3.10), we obtain

$$
\begin{aligned}
H(t) & \geq \sqrt{\Gamma(1-\alpha)} w\left(t_{1}\right)+\sqrt{\Gamma(1-\alpha)} \int_{t_{1}}^{t} \frac{1}{g(s)}\left(H(s)-\frac{p(s) g(s)}{2 \sqrt{\Gamma(1-\alpha)}}\right)^{2} d s \\
& -\frac{1}{4 \sqrt{\Gamma(1-\alpha)}} \int_{t_{1}}^{t}\left[p^{2}(s) g(s)+\frac{\left(g^{\prime}(s)\right)^{2}}{g(s)}-2 p(s) g^{\prime}(s)-4 K \Gamma(1-\alpha) g(s) q(s)\right] d s \\
& +\frac{g^{\prime}(t)}{2 \sqrt{\Gamma(1-\alpha)}} .
\end{aligned}
$$

Now, from (3.6) there exists $t_{2} \geq t_{1}$ such that

$$
H(t)>\sqrt{\Gamma(1-\alpha)} \int_{t_{1}}^{t} \frac{1}{g(s)}\left(H(s)-\frac{p(s) g(s)}{2 \sqrt{\Gamma(1-\alpha)}}\right)^{2} d s .
$$

Let

$$
Q(t)=\sqrt{\Gamma(1-\alpha)} \int_{t_{1}}^{t} \frac{1}{g(s)}\left(H(s)-\frac{p(s) g(s)}{2 \sqrt{\Gamma(1-\alpha)}}\right)^{2} d s .
$$

Then, using the fact that $p(t)<0$, we have $H(t)>Q(t)>0$. Differentiating (3.11) gives

$$
\begin{aligned}
Q^{\prime}(t) & =\sqrt{\Gamma(1-\alpha)} \frac{1}{g(t)}\left(H(t)-\frac{p(t) g(t)}{2 \sqrt{\Gamma(1-\alpha)}}\right)^{2} \\
& \geq \sqrt{\Gamma(1-\alpha)} \frac{1}{g(t)}\left(Q(t)-\frac{p(t) g(t)}{2 \sqrt{\Gamma(1-\alpha)}}\right)^{2}
\end{aligned}
$$




$$
>\sqrt{\Gamma(1-\alpha)} \frac{1}{g(t)} Q^{2}(t)
$$

and so we have

$$
\frac{\sqrt{\Gamma(1-\alpha)}}{g(t)}<\frac{Q^{\prime}(t)}{Q^{2}(t)} .
$$

Integrating both of sides of this inequality from $t_{2}$ to $t$, we obtain

$$
\int_{t_{2}}^{t} \frac{\Gamma(1-\alpha)}{g(s)} d s<\frac{1}{Q\left(t_{2}\right)}-\frac{1}{Q(t)}<\frac{1}{Q\left(t_{2}\right)}
$$

which contradicts (3.5) and completes the proof of the theorem.

Example 1. Consider the fractional differential equation, for $t>0$,

$$
\left(D_{0+}^{1+\alpha} y\right)(t)-\frac{1}{t^{2}}\left(D_{0+}^{\alpha} y\right)(t)+\left(1+\frac{1}{t^{6}}\right) \int_{0}^{t}(t-s)^{-\alpha} y(s) d s=0,
$$

with $\alpha=1 / 2$. Here, $p(t)=-1 / t^{2}, q(t)=1+1 / t^{6}$, and $f(u)=u$. Then we have $K=1$, and $\Gamma(1-\alpha)=\Gamma(1 / 2)=\sqrt{\pi}$. Let $t_{0}=1$, thus condition (3.1) becomes

$$
\begin{aligned}
& \lim _{t \rightarrow \infty}\left[\frac{1}{4 \Gamma(1-\alpha)} \int_{t_{0}}^{t}\left(4 \Gamma(1-\alpha) K q(s)-p^{2}(s)\right) d s\right] \\
& =\lim _{t \rightarrow \infty}\left[\frac{1}{4 \sqrt{\pi}} \int_{1}^{t}\left(4 \sqrt{\pi}\left(1+\frac{1}{s^{6}}\right)-\frac{1}{s^{4}}\right) d s\right] \\
& =\lim _{t \rightarrow \infty}\left[\left.\frac{1}{4 \sqrt{\pi}}\left(4 \sqrt{\pi} s-\frac{4 \sqrt{\pi}}{5 s^{5}}+\frac{1}{3 s^{3}}\right)\right|_{1} ^{t}\right]=\infty .
\end{aligned}
$$

So every solution of (3.12) is oscillatory by Theorem 1

Example 2. Consider the fractional differential equation

$$
\begin{aligned}
& \left(D_{0+}^{1+\alpha} y\right)(t)-\frac{1}{t}\left(D_{0+}^{\alpha} y\right)(t) \\
& +\left(1+\frac{1}{t^{2}}\right) \frac{1}{\sqrt{\pi}} \exp \left(\int_{0}^{t}(t-s)^{-\alpha} y(s) d s\right) \int_{0}^{t}(t-s)^{-\alpha} y(s) d s=0,
\end{aligned}
$$

with $\alpha=1 / 2$. Here, $p(t)=-1 / t, q(t)=1+1 / t^{2}$, and $f(u)=\frac{1}{\sqrt{\pi}} e^{u} u$. Then, we have $K=1 / \sqrt{\pi}$, and $\Gamma(1-\alpha)=\Gamma(1 / 2)=\sqrt{\pi}$. If we take $t_{0}=1$, and $g(t)=t$, 
then

$$
\lim _{t \rightarrow \infty} \int_{t_{0}}^{t} \frac{\Gamma(1-\alpha)}{g(s)} d s=\lim _{t \rightarrow \infty} \int_{1}^{t} \frac{\sqrt{\pi}}{s} d s=\infty
$$

and condition (3.6) becomes

$$
\begin{aligned}
& \lim _{t \rightarrow \infty}\left[-\frac{1}{4 \pi^{1 / 4}} \int_{1}^{t}\left\{\frac{1}{s}+\frac{1}{s}+\frac{2}{s}-4 \frac{1}{\sqrt{\pi}} \sqrt{\pi} s\left(1+\frac{1}{s^{2}}\right)\right\} d s+\frac{1}{2 \pi^{1 / 4}}\right] \\
& =\lim _{t \rightarrow \infty}\left[-\frac{1}{4 \pi^{1 / 4}} \int_{1}^{t}\{-4 s\} d s+\frac{1}{2 \pi^{1 / 4}}\right] \\
& =\lim _{t \rightarrow \infty}\left[\left.\frac{1}{2 \pi^{1 / 4}} s^{2}\right|_{1} ^{t}+\frac{1}{2 \pi^{1 / 4}}\right]=\infty .
\end{aligned}
$$

We see that the hypotheses of Theorem 2 are satisfied and so all solutions of (3.13) are oscillatory.

\section{REFERENCES}

[1] H. K. Abdullah, "A note on the oscillation of second order differential equations," Czech. Math. J., vol. 54, no. 4, pp. 949-954, 2004, doi: 10.1007/s10587-004-6443-3.

[2] G. A. Anastassiou, Fractional Differentiation Inequalities. NewYork: Springer, 2009.

[3] D.-X. Chen, "Oscillatory behavior of a class of fractional differential equations with damping," U.P.B. Sci. Bull.,Series A, vol. 75, no. 5, pp. 107-118, 2013.

[4] S. R. Grace, "On the asymptotic behavior of positive solutions of certain integral equations," Appl. Math. Lett., vol. 44, pp. 5-11, 2015, doi: 10.1016/j.aml.2014.12.005.

[5] S. R. Grace, R. P. Agarwal, and J. R. Graef, "Oscillation criteria for certain third order nonlinear difference equations," Appl. Anal. Discrete Math., vol. 3, no. 1, pp. 27-38, 2009, doi: 10.2298/AADM0901027G.

[6] S. R. Grace, R. P. Agarwal, P. J. Y. Wong, and A. Zafer, "On the oscillation of fractional differential equations," Fract. Calc. Appl. Anal., vol. 15, no. 2, pp. 222-231, 2012, doi: 10.2478/s13540-0120016-1.

[7] A. A. Kilbas, H. M. Srivastava, and J. J. Trujillo, Theory and Applications of Fractional Differential Equations. Amsterdam: Elsevier Science B.V, 2006.

[8] I. Podlubny, Fractional Differential Equations, ser. Mathematics in Science and Engineering. New York: Academic Press, 1999.

[9] P. Prakash, S. Harikrishnan, J. J. Nieto, and J.-H. Kim, "Oscillation of a time fractional partial differential equation," Electron. J. Qual. Theory Differ. Equ., vol. 2014, no. 15, pp. 1-10, 2014, doi: 10.14232/ejqtde.2014.1.15.

[10] S. G. Samko, A. A. Kilbas, and O. I. Marichev, Fractional Integrals and Derivatives, ser. Theory and Applications. Amsterdam: Gordon and Breach, 1993.

[11] E. Tunç, "A note on the oscillation of second order differential equations with damping," J. Comput. Anal. Appl., vol. 12, no. 2, pp. 444-453, 2010.

[12] E. Tunç, "Oscillation results for even order trinomial functional differential equations with damping," Appl. Appl. Math.: An International Journal (AAM), vol. 9, no. 2, pp. 696-717, 2014. 
[13] J. Yang, A. Liu, and T. Liu, "Forced oscillation of nonlinear fractional differential equations with damping term," Adv. Difference Equ., vol. 2015, no. 1, pp. 1-7, 2015, doi: 10.1186/s13662-0140331-4.

\section{Authors' addresses}

\section{Ercan Tunç}

Gaziosmanpasa University, Department of Mathematics, Faculty of Arts and Sciences, 60240, Tokat, Turkey

E-mail address: ercantunc72@yahoo.com

\section{Osman Tunç}

Yüzüncü Yil University, 7A-8, 65080, Van, Turkey

E-mail address: osmantunc89@gmail.com 